\title{
Simultaneous motion perception along multiple attributes: A new class of stimuli
}

\author{
THOMAS V. PAPATHOMAS \\ AT\& T Bell Laboratories, Murray Hill, New Jersey \\ and \\ ANDREI GOREA \\ Université René Descartes, Paris, France \\ and CNRS, Paris, France
}

\begin{abstract}
This paper describes a novel class of visual stimuli that have been created to study stroboscopic motion perception along multiple attributes. The stimuli are composed of discrete elements, each of which can be characterized by an arbitrary number of attributes (color, luminance, spatial frequency, binocular disparity, etc.). There are two choices for the spatiotemporal arrangement of each attribute: to elicit either unambiguous (unidirectional) motion perception or ambiguous (bidirectional) motion perception. This affords the unique ability to elicit simultaneous motion perception in opposing directions from identical stimuli. The prevailing direction depends on the relative strength of the attributes' contribution to the motion mechanisms (for short frame duration), or on the particular attribute being attended to (for long frame duration). Thus, this class of stimuli opens up the possibility of isolating specific motion mechanisms in the visual system.
\end{abstract}

The problem of identifying the visual attributes (luminance, color, contrast, form, etc.) that contribute to apparent motion perception and of assessing the relative strength with which each attribute activates motion mechanisms has received increasing attention over the past two decades. In these studies, apparent motion is elicited by the spatial matching of image elements over time, that is, across frames presented in rapid succession. The path that an object takes in apparent motion depends on how the visual system matches the attributes of the object at different places and at different times, a process called correspondence matching. In addition to the interframe displacement, $\Delta x$, between corresponding points, the duration, $T$, of each frame, and the time delay, $\Delta t$, between successive frames, the path also depends on the relative strength with which the matched attribute(s) of the object activate(s) the motion mechanisms in the visual system.

Researchers have manipulated all these parameters in an effort to assess the relative motion strength of various attributes. Navon (1976), for example, used stimuli of different shapes arranged in a circle that rotated from frame to frame in either direction with equal probability; this ambiguity was resolved in the stimuli by matching the figural attribute to produce motion in a preferred direction. Navon observed, however, that figural identity was not a strong "token" for carrying motion, a result that

\footnotetext{
We are grateful to Ted (E. H.) Adelson, Patrick Cavanagh, Bart Farell, John Krauskopf, and Jan van Santen for useful comments and discussions. We are particularly indebted to Bela Julesz for his support and encouragement. Please address correspondence to Thomas V. Papathomas, AT\&T Bell Laboratories, 600 Mountain Avenue, Murray Hill, NJ 07974-2070.
}

was observed earlier by Kolers and Pomerantz (1971) via a different technique. Kolers (1972) presented additional experiments supporting the same conclusion, as did Hochberg and Brooks (1974) by investigating the roles of $\Delta x$, $T$, and shape (see also Hochberg, 1978). Sperling (1976) obtained a function of the strength of the perceived motion as a function of $\Delta x$ and $\Delta t$, and later Burt and Sperling (1981) developed a generalized methodology allowing the study of time, distance, and attribute tradeoffs in apparent motion, and derived a model based on their observations. The latter methodology was the first to offer a quantitative measure of an attribute's ability to elicit motion, by manipulating $\Delta x$ in multiple-path stimuli.

The techniques of Burt and Sperling (1981) and similar ones were employed to assess the contribution to motion perception of orientation and shape (Green, 1986; Sperling, van Santen, \& Burt, 1985; Ullman, 1979, 1980; Watson, 1986), size and/or spatial frequency (Green, 1986; Ullman, 1980; Watson, 1986), luminance (Adelson \& Bergen, 1985; Anstis, 1980; Cavanagh, Anstis, \& Mather, 1984; Ullman, 1979; van Santen \& Sperling, 1985; Watson \& Ahumada, 1983, to name a few recent reports), phase (Green, 1986), contrast (Pantle \& Sekuler, 1969; Thompson, 1982), and binocular disparity (Green \& Odom, 1986). The general conclusion is that all of the above attributes except phase carry motion. The apparent contradiction between experimenters who reported that orientation does carry motion (Green, 1986; Ullman, 1979) and those who concluded that it does not (Burt \& Sperling, 1981; Watson, 1986) can be explained in terms of the spatial characteristics of their stimuli. A developing domain of interest concerns the contribution to motion perception of purely chromatic inputs. Many reports 
concur that equiluminant chromatic input is much less effective than luminance input for producing a motion response (Cavanagh \& Anstis, 1986; Cavanagh \& Favreau, 1985; Cavanagh, Tyler, \& Favreau, 1984; Moreland, 1980; Ramachandran \& Gregory, 1978; Troscianko, 1987). Parenthetically, a wide variety of techniques were used, and some were quite different from those cited earlier in this paragraph.

The common elements in most of the studies that used apparent-motion stimuli with discrete elements are (1) the use of $\Delta x, T$, and $\Delta t$ as the independent variables determining the "strength units" and permitting the assessment of the "relative strength" of the various attributes, and (2) the matching of one single attribute at a time, while all others are not varied and thus are matched ambiguously, that is, simultaneously to the left and to the right. Thus, these techniques suffer from the limitation that two attributes can be compared only indirectly, that is, based on trials that are conducted at two separate times. There are a few exceptions in which investigators were able to effect a direct comparison of attributes. Ramachandran, Ginsburg, and Anstis (1983) designed an experiment that indicated that the direction of movement is determined by low spatial frequencies, rather than by the orientation of edges. Their stimuli, however, cannot be generalized to cover systematically other attributes. Cavanagh, Tyler, and Favreau (1984) employed simultaneously displayed moving sine-wave gratings defined by luminance and chrominance, but these were spatially separated. Kooi, De Valois, and Wyman (1988) used the attributes of spatial frequency, contrast, and chrominance in studying the movement of plaids formed by two sinewave gratings.

In contrast to most previous studies that involve discrete elements (i.e., not gratings), our new class of visual stimuli allows the direct comparison of the contribution of any two attributes to motion perception. The characteristics of the stimuli are the following: (1) in the canonical form, we use a "balanced" $\Delta x$ by having equal displacements to the left and to the right (however, unequal displacements can also be used); (2) we selectively match one or more attributes at a time while leaving the remaining ones unmatched; and (3) when two or more attributes are matched, there are two options: either to match them along the same direction (concurrent matching) or along different ones (competitive matching). This flexibility in manipulating the stimuli makes it possible to (a) isolate specific motion mechanisms in the absence of stimulation of any other mechanisms, (b) assess the summation index for motion strength when two or more attributes are matched concurrently, and (c) directly assess the relative strength of pairs of attributes by using competitive matchings to determine which of them prevails in determining the perceived direction of motion.

Furthermore, the method is not restricted to only two attributes; one combination of attributes may be compared with another combination of attributes with respect to the strength of the induced motion. Finally, our concept can be adapted to be used with other investigators' sets of stimuli.

\section{A NEW CLASS OF STIMULI}

\section{The Concept}

Consider the configuration of Figure 1a. Each row represents the spatial arrangement of dots at a given instant $t_{n}$. The dots are equidistant along $x$ with period $x_{0}$. In the next time frame, $t_{n+1}$, the arrangement is replaced by that of the next row. The interframe time interval, $\Delta t$, is fixed. Figure la shows this motion sequence schematically in the $x-t$ space (Adelson \& Bergen, 1985). Motion is elicited to the right (see path $d_{R}$ ) because $\Delta x<$ $x_{0} / 2$ (if $\Delta x>x_{0} / 2$, leftward motion would be observed).

By contrast, consider the stimulus of Figure $1 b$, where $\Delta x=x_{0} / 2$. It is clear that this sequence will elicit ambiguous motion if all the elements are identical, because matchings along paths $d_{L}$ and $d_{R}$ are equally probable. $\mathrm{Ob}-$ servers are expected to report leftward motion as frequently as rightward motion. However, this ambiguity can be resolved by assigning different values to an attribute $A$ of the elements, as shown by Burt and Sperling (1981). To illustrate, if $A$ is binocular disparity, two values $A_{1}$ and $A_{2}$ can be assigned to the elements so as to elicit motion to the right, as in Figure 1b. Green and Odom (1986) used a variant of this stimulus, arranged in a circle, to demonstrate that disparity is a token for motion. Indeed, motion would be elicited with the stimulus of Figure $1 \mathrm{~b}$ only if attribute $A$ is a token for the perception of movement, and such stimuli can be used as a test for other attributes (color, orientation, spatial frequency, etc.). Notice that the horizontal period in Figure $1 \mathrm{~b}$ is now $2 x_{0}$, a fact that was used to resolve the ambiguity.

(a)

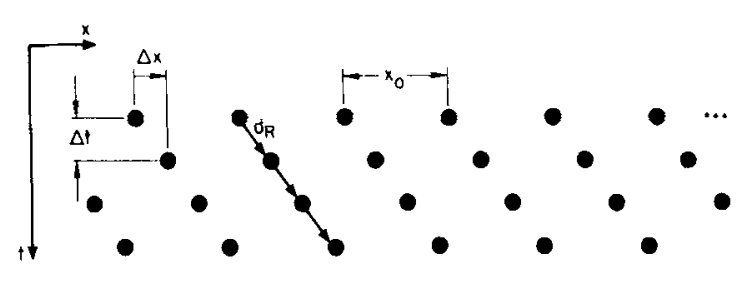

(b)

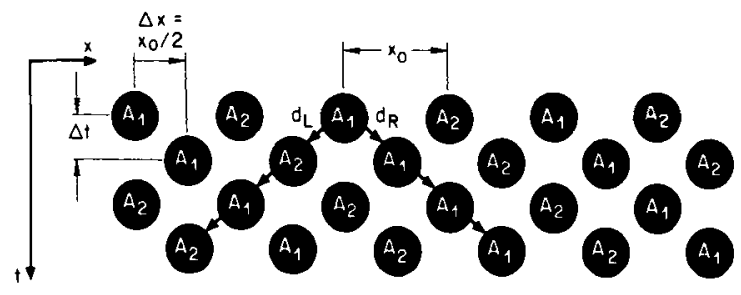

Figure 1. The spatiotemporal arrangement of dots in (a) produces rightward motion along path $d_{R}$. In (b), paths $d_{L}$ and $d_{R}$ are equally likely, resulting in ambiguous direction of motion for identical elements. However, when two different values, $A_{1}$ and $A_{2}$, of an attribute $A$ are assigned to the dots as shown, rightward motion ensues along path $d_{R}$ if the attribute $A$ is a token for motion perception. 
Our stimuli offer a significant improvement over those of Figure $1 \mathrm{~b}$. They allow an arbitrary number of attributes $A, B, \ldots, Z$ to be used, rather than only one. Furthermore, they offer the flexibility to arrange the values of each attribute in the $x-t$ plane so as to (1) produce motion to the left, (2) produce motion to the right, or (3) produce ambiguous motion, independently of the other attributes. This can result in a "pandemonium" of motion, in which the prevailing percept depends on several factors.

\section{Multiattribute Stimuli}

To make matters concrete, Figure 2a illustrates a member of the new class of stimuli. The elements occupy discrete positions in the $x-t$ plane, and they may fill the space of the corresponding "block" partially or entirely. The width of each block is $p$ and the separation between blocks is $q$, resulting in an interelement distance $x_{0}=p+q$, measured from center to center. The values of the discretized spatial and temporal variables $j$ and $i$ are shown along the top row and along the left column, respectively. Only three attributes $A, B$, and $C$ are shown for each element for simplicity. Let $N$ be the number of different values for each attribute. We have arbitrarily chosen $N=3$ here, so that each attribute may assume three distinct values, indicated by subscripts on Figure 2a. Next, we examine the effect of this spatiotemporal arrangement on the perception of motion.

Suppose, for this paragraph only, that attribute $C$ is fixed at level $C_{0}$; that is, consider Figure $2 \mathrm{a}$ with all the subscripts of $C$ equal to zero. It must be clear that this uniform matching of $C$ has no influence in eliciting motion. If attribute $A$ is a token for motion, then this stimulus will elicit unambiguous rightward movement along path $d_{A R}$ because attribute $A$ is matched along it; indeed, the value of $A$ remains fixed along paths parallel to $d_{A R}$, while its value changes cyclically from $A_{1}$ to $A_{0}$ to $A_{2}$ along paths parallel to $d_{A L}$, the leftward path. On the other hand, attribute $B$ must produce unambiguous leftward motion along path $d_{B L}$ for identical reasons, provided that attribute $B$ is a "carrier" for motion. When frames 0 to 5 are shown in sequence, three possible percepts can ensue. (1) In the case of perceived motion to the right, there are two possibilities: attribute $A$ is a token for motion, but $B$ is not, or both $A$ and $B$ are tokens for motion, and $A$ elicits stronger movement perception than $B$. (2) If there is unambiguous motion to the left, we have the same two possibilities as in case 1 , but the roles of attributes $A$ and $B$ are reversed. Finally, (3) if there is no coherent motion in either direction, again there are two explanations:

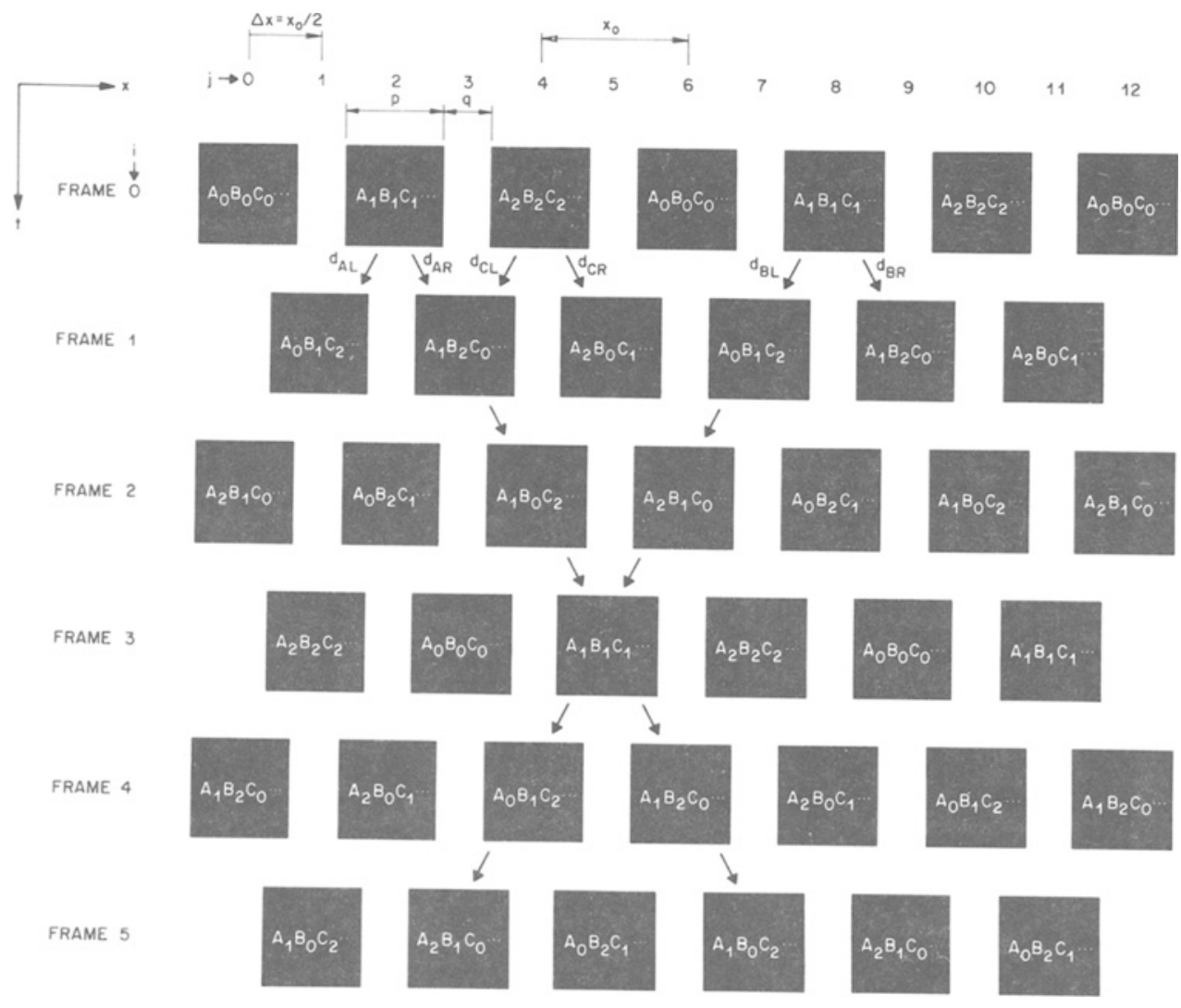

Figure 2. (a) A schematic diagram in the $x-t$ plane of a typical member of the new class of stimuli. Only three attributes, $A, B$, and $C$, are shown for clarity. Notice that attributes $A$ and $B$ are matched to produce motion to the right and left, respectively, and attribute $C$ to produce ambiguous motion. (Figure 2 continued next page $\rightarrow$ ) 
(2b)

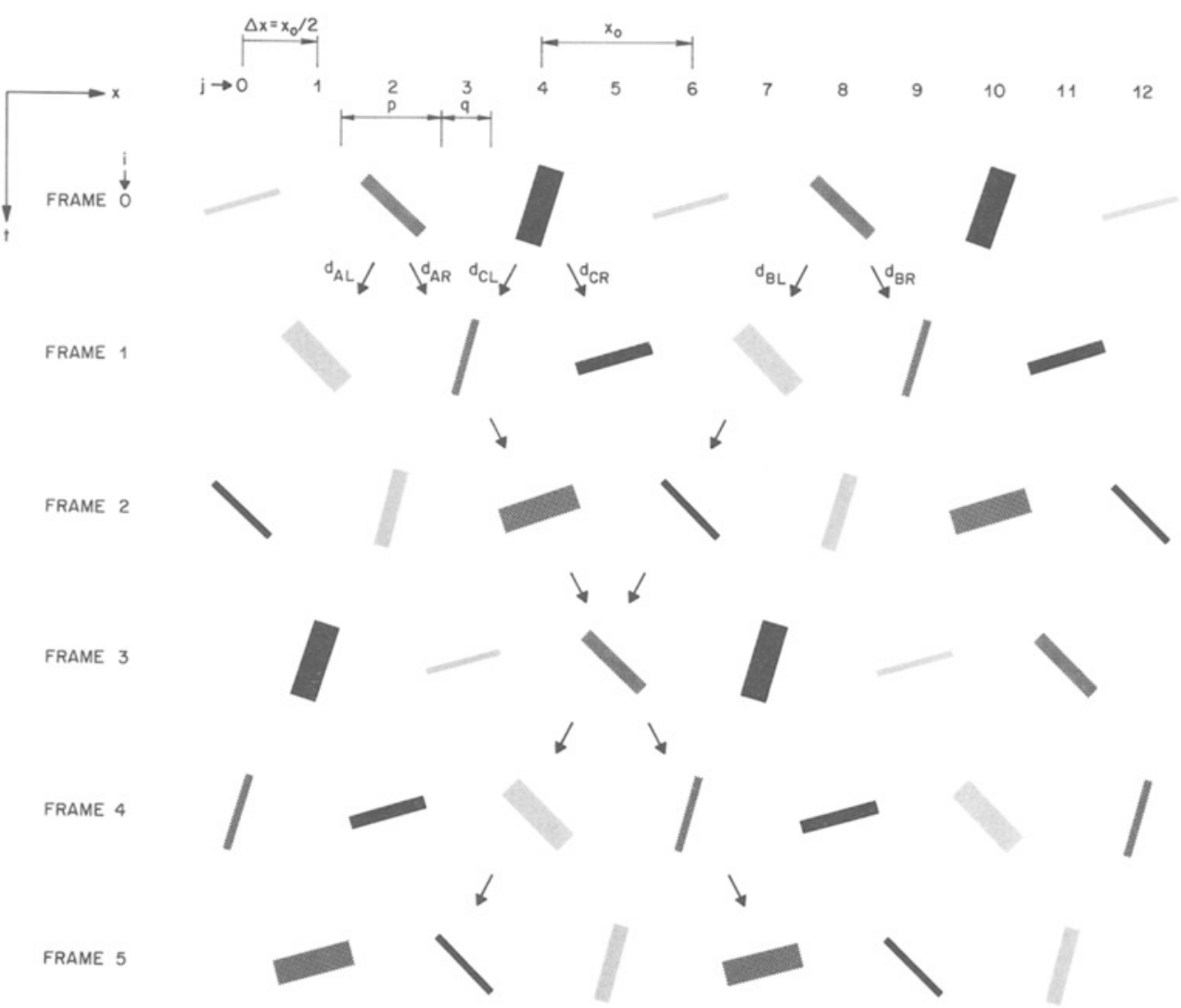

(2c)
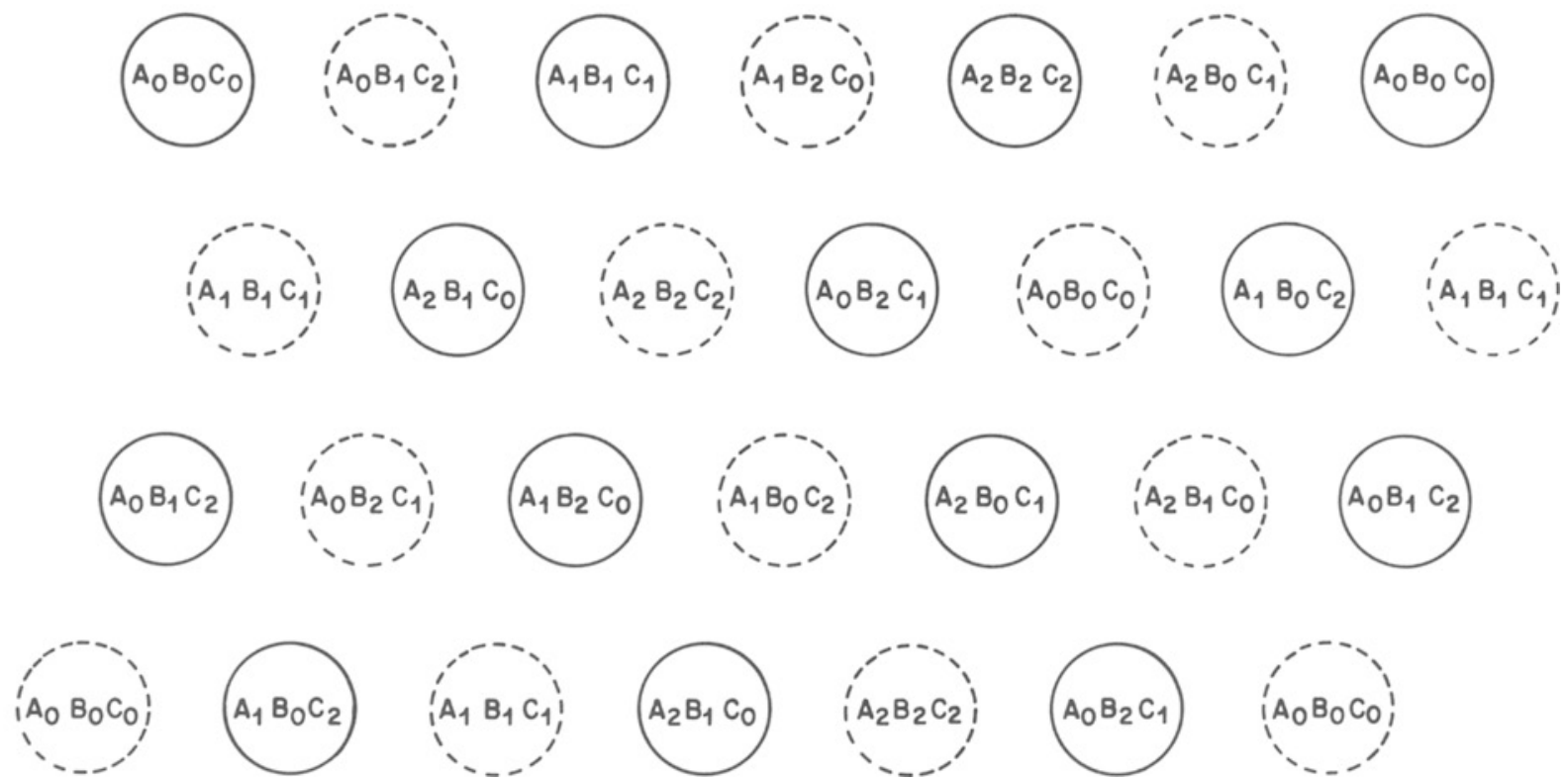

(Figure 2, continued.) (b) A specific instance of (a), where $A, B$, and $C$ are luminance, orientation, and size, respectively. (c) The first two frames of a multirow display. Frames 0 and 1 are indicated by solid and dashed circles, respectively. Attributes $A, B$, and $C$ are matched to produce rightward, leftward, and ambiguous motion, respectively. 
neither attribute $A$ nor attribute $B$ is a carrier for motion, or both $A$ and $B$ can carry motion, but their "strengths" in eliciting movement are equal and their effects cancel each other. Of course, if we want to test whether a given attribute of interest is a carrier of motion, we can use it by itself, as shown in Figure 1b.

In the preceding paragraph, it was assumed that attribute $C$ was identical for all the elements in each frame. Now, let us consider the spatiotemporal arrangement of Figure $2 \mathrm{a}$, in which the value of $C$ varies cyclically along both the leftward $\left(d_{C L}\right)$ and the rightward $\left(d_{C R}\right)$ paths. For this pattern, attribute $C$ is described as unmatched. It is obvious that, even if $C$ is a token for motion, this arrangement for attribute $C$ cannot produce unambiguous (unidirectional) motion perception. Thus, if the sequence of Figure 2a elicits unambiguous motion to the left or to the right, this must be due to attributes $A$ or $B$ and cannot be attributed to the influence of attribute $C$. Also, if incoherent motion is elicited without any preference for either direction, no conclusions can be drawn about the influence of $C$ to motion. The reason we allow this unmatched arrangement, in addition to the uniform matching, of an attribute is explained in the concluding paragraph of Isolation of Specific Motion Mechanisms, below.

To illustrate, let $A$ be local luminance, that is, the luminance of each picture element (pixel) on the display, such that $A_{0}=$ light gray, $A_{1}=$ dark gray, and $A_{2}=$ black. Let $B$ be the orientation of each element, where each element is a bar of length $p$, with $B_{0}=15^{\circ}, B_{1}=$ $135^{\circ}$, and $B_{2}=255^{\circ}$. Finally, let $C$ be the size (width) of each bar so that $C_{0}=s, C_{1}=2 s$, and $C_{2}=3 s$. The selection of these attributes is arbitrary and artificial. The reason for choosing them is that it is easy to illustrate the resulting stimulus, as shown in Figure $2 \mathrm{~b}$. (We could have selected spatial frequency, contrast, color, binocular disparity, etc., but it would have been extremely difficult to produce the stimulus in print.) Notice the patterns generated in Figure $2 \mathrm{~b}$, suggesting rightward motion for luminance (attribute $A$ ), leftward motion for orientation (attribute $B$ ), and no consistent motion for size (attribute $C$ ). Also, notice that the elements occupy only a portion of the defining "blocks" of Figure 2a.

\section{Automatic Generation of Stimuli}

To generate these stimuli on a computer graphics work station for psychophysical experiments, it is desirable to have formulas for the subscripts of each attribute in terms of the discretized spatial and temporal parameters $j$ and $i$, respectively, and the number of distinct values, $N$, that each attribute is allowed to take. We shall adopt the following notation: If we desire to arrange an attribute $U$ in the $x-t$ plane so as to produce leftward, rightward, or ambiguous motion, we shall assign to it subscript $l$ (for left), $r$ (for right), and $a$ (for ambiguous). The formulas are:

$$
\begin{gathered}
l=[(j+1) \%(2 N)] / 2, \\
r=[(j-i) \bmod (2 N)] / 2,
\end{gathered}
$$

and

$$
a=(-j) \bmod N,
$$

where $(x \% y)$ means the integer remainder of the division of $x$ by $y$ and mod is the modulo function, defined by

$$
x \bmod y= \begin{cases}x \% y & \text { for } x \geq 0 \\ y+(x \% y) & \text { for } x<0\end{cases}
$$

The subscripts $r$ and $l$ are defined only at $(i, j)$ when $(i+j)$ is even (see Figure 2a). To illustrate, the subscript for attribute $B$ of Figure 2a (leftward motion) at $i=3, j=5$ is $l=[(3+5) \% 6] / 2=1$; the subscript for $A$ (rightward motion) at $i=4, j=10$ is $r=[(10-4) \bmod 6] / 2=0$; and that for $C$ (ambiguous motion) at $i=1, j=5$ is $a=(-5)$ $\bmod 3=1$, as indeed is the case in Figure $2 a$.

\section{USING THE STIMULI}

\section{Inherent Properties}

This class of stimuli exhibits two desirable properties, which can be verified either by inspecting Figure $2 \mathrm{a}$ or by using Equations 1-3:

Property 1 (Periodicity along $x$ ). The stimuli are periodic along $x$ with a period $N$. This fact can be taken advantage of for the purpose of generating the stimuli faster on the computer.

Property 2 (Periodicity along $t$ ). The stimuli are periodic in $t$ with a period $P=2 N$. This can be utilized in creating motion sequences of arbitrarily long duration by cycling through the $2 N$ rows.

Additionally, the same set of $2 N$ rows, stored in the computer's graphics memory, can be used to produce either leftward or rightward motion due to the very same attribute by "playing" the frames forward (as shown in Figure 2a) or backward (i.e., reversing the intended flow of time, showing frame 5 first, then frame 4 , etc.). There is no need to use only one row per frame. For example, Figure 2c portrays a situation in which four rows are simultaneously displayed during frame 0 (solid circles). During frame 1 , each row is replaced by its successor row (dashed circles), as dictated by Equations 1-3. In this case, attributes $A$ and $B$ are matched to elicit rightward and leftward motion, respectively, whereas $C$ is matched cyclically, producing ambiguous motion. Random horizontal shifts are assigned to each row to avoid the formation of regular patterns.

Finally, these stimuli provide a test for whether a specific attribute is a token for motion. One can design stimuli in which the attribute is matched to produce movement perception and then try these stimuli to see if indeed such perception is elicited.

\section{Typical Stimuli and Experiments}

We have created a variety of stimuli that are members of this class. In most cases, we used two attributes $A$ and $B$, with $N=2$ or $N=3$. For each of the following cases, three sequences were generated: (1) $A$ was arranged to produce unambiguous motion and $B$ to produce ambigu- 
ous motion, (2) the roles of $A$ and $B$ were interchanged, and (3) $A$ and $B$ produced unambiguous but simultaneously present opposing motions. (An additional condition, not discussed here, consisted in arranging attributes $A$ and $B$ to produce unambiguous motion in the same direction.) For the purposes of our research, we always selected orientation to be attribute $B$ and we have worked with the following five attributes, one at a time, to complete the pair.

1. $A=$ color. We conducted several experiments under nonequiluminant and equiluminant conditions, in which we obtained evidence that chromatic contrast is a stronger motion carrier than reported from previous studies. Moreover, these experiments suggested the existence of three mechanisms: a luminance, a pure chromatic, and a combination of chromatic and luminance mechanisms which provide motion information (Gorea \& Papathomas, 1988c).

2. $A=$ luminance. Experimenting with this and the preceding subset of stimuli, we gathered data that indicate that motion mechanisms can establish correspondences across distinct orientation and luminance attributes, but cannot do so across distinct color attributes under equiluminant conditions (Gorea \& Papathomas, 1988b).

3. $A=$ polarity. The background here is gray and $N=2$. $\mathrm{A}_{1}=$ black, $A_{2}=$ white, and $B_{1}=45^{\circ}, B_{2}=135^{\circ}$ were used (as well as $B_{1}=0^{\circ}, B_{2}=90^{\circ}$ ).

4. $A=$ binocular disparity. We used dynamic randomdot stereograms (DRDS) with $N=2, A_{1}=+15$ min of arc, $A_{2}=-15 \mathrm{~min}$ of arc, $B_{1}=45^{\circ}$, and $B_{2}=135^{\circ}$ (Papathomas, Gorea, Julesz, \& Chang, 1988).

5. $A=$ spatial frequency. Here we used circular Gabor patches (Caelli, 1986; Daugman, 1984) against a background of uniform luminance with $A_{1}=6$ cycles per degree (cpd), $A_{2}=10 \mathrm{cpd}$, and $B_{1}=45^{\circ}, B_{2}=135^{\circ}$ (see Figure 3).

In cases 3-5, the main observation was that motion mechanisms can match the attribute $A$ across orientation channels much more easily than they can match orienta- tion across the channels defined by polarity, disparity of different sign, or spatial frequency (Gorea \& Papathomas, 1988a).

The above results were obtained from experiments in which two to five frames were shown in each trial, and the frame duration was short $(33.33 \mathrm{msec})$. We also used the temporal periodicity of the stimuli to produce arbitrarily long cyclic sequences with longer frame durations $(100-200 \mathrm{msec})$ in which attributes $A$ and $B$ "moved" in opposite directions, using the same combination of attributes as before. Most subjects were able to attend to one attribute and perceive motion in one direction and then voluntarily shift to the other attribute and see motion in the opposite direction. This shift was facilitated by smooth-pursuit eye movements. In the case of color versus orientation and luminance versus orientation, some subjects were able to perceive both (opposing) motions simultaneously, thus "decomposing" the elements into their constituent attributes.

\section{Isolation of Specific Motion Mechanisms}

To illustrate how a specific motion mechanism can be isolated by judicious choices of stimulus configuration, consider the attributes of color and orientation. Figure 4a, in which black and white elements denote two different but equiluminant colors, shows an arrangement in which the color of individual elements is matched, but not the orientation. The converse is true for Figure $4 \mathrm{~b}$, whereas in Figure $4 \mathrm{c}$ the orientation is matched, while color, being uniform, is matched in both directions. We start with the reasonable assumptions (1) that there are both chromatic and achromatic motion pathways (Michael, 1985), (2) that both luminance and color pathways consist of both orientation-tuned and orientation-nonselective units (Zeki, 1983), and (3) that these units provide the input to Reichardt-type motion detectors (van Santen \& Sperling, 1985). For brevity's sake, we use the following notation: $\mathrm{C}-\mathrm{O}$ and $\mathrm{C}-\mathrm{nO}$ denote chromatic cells that are orienta-

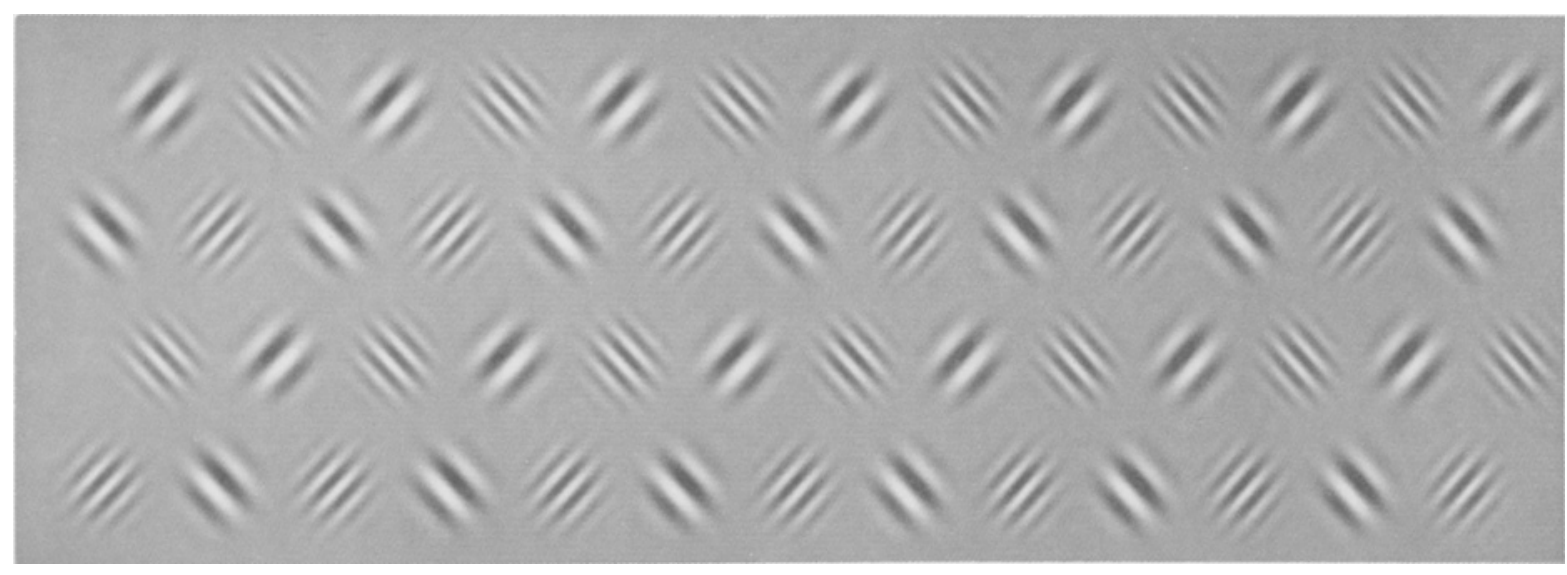

Figure 3. A stimulus that was used in an experiment to compare the relative strength of orientation and spatial frequency as carrier of motion. The spatial variahle $x$ extends horizontally and time grows downward. Notice that the directions of motion elicited by matching orientation (rightward) and spatial frequency (leftward) oppose each other. 


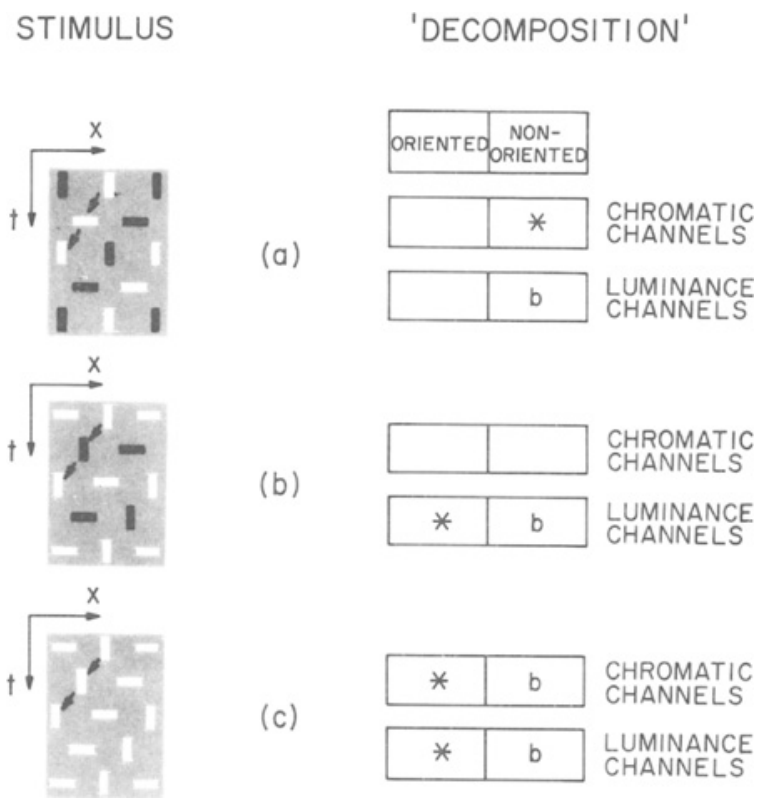

Figure 4. The left column shows three motion stimuli in which color and orientation are the selected attributes with $N=2$. Black and white bars denote two differently colored, equiluminant elements. The right column shows the corresponding tables containing the activation status of specific motion channels. A blank entry in the table denotes no activation, " $\mathrm{b}$ " stands for balanced activation, and an asterisk indicates unambiguous stimulation. The arrows indicate the path favored by the spatiotemporal matching. In (a), color is matched to elicit motion to the left while orientation is unmatched in both directions. In (b), orientation is matched while color is unmatched. Orientation is also matched in (c), while color, being constant, is matched in both directions.

tion tuned and orientation nonselective, respectively. The luminance cells $\mathrm{L}-\mathrm{O}$ and $\mathrm{L}-\mathrm{nO}$ are similarly defined.

Since orientation is not matched in either direction in Figure $4 \mathrm{a}$, the $\mathrm{C}-\mathrm{O}$ and $\mathrm{L}-\mathrm{O}$ motion channels are not activated, hence the blank entries in the table of the right column of Figure 4a. The $\mathrm{L}-\mathrm{nO}$ channels are activated equally in both directions, resulting in ambiguous perception, because the stimulus of Figure $4 \mathrm{a}$ is drift-balanced (Chubb \& Sperling, 1987) with respect to luminance due to the equiluminance of the elements (entry " $b$ " in the table of Figure 4a). Since the matched attribute in Figure $4 \mathrm{a}$ is color, not orientation, this stimulus activates unambiguously the $\mathrm{C}-\mathrm{nO}$ channels, as denoted by the asterisk in the table of Figure 4a. This is an example of direct selective isolation of a specific motion channel. Another example is offered by Figure 4b, where the L-O channels are selectively stimulated.

In addition to direct isolation, we can use combinations of experiments to indirectly infer the strength of specific motion channels. For example, we compared the results of experiments using the stimuli of Figure $4 b$ with those using the stimuli of Figure $4 \mathrm{c}$, in which the $\mathrm{C}-\mathrm{O}$ and $\mathrm{L}-\mathrm{O}$ channels are activated while the $\mathrm{C}-\mathrm{nO}$ and $\mathrm{L}-\mathrm{nO}$ mechanisms have balanced activity. By comparing the tables of Figures $4 b$ and $4 c$, we notice that the only different unambiguous entry is $\mathrm{C}-\mathrm{O}$. Thus, comparing the motion de- tection performances obtained with stimuli $4 b$ and $4 c$ enabled us to conclude that oriented color channels contribute to motion perception (Gorea \& Papathomas, 1988c).

Incidentally, Figures $4 \mathrm{~b}$ and $4 \mathrm{c}$ can serve to illustrate one of the main differences between the stimuli of other researchers and those presented in this paper. Figure 4c, or similar versions (see, e.g., Figure 4 in Burt \& Sperling, 1981, and Figure 1 in Green, 1986), have been employed to isolate the role of one attribute (in this case orientation), while all other attributes (in this case color, length, width, etc.) are kept fixed; this is what we call "matching one attribute (orientation) within other attributes." By contrast, in the stimulus shown in Figure 4b, orientation is matched to produce motion to the left, while another attribute (in this case color) varies so as not to contribute unambiguous motion. This is what we call "matching one attribute across another attribute," and it opens up new possibilities in studying motion mechanisms. Parenthetically, we also have produced stimuli in which the value of the second attribute varies randomly in space and time. Again, the "moving" attribute is matched across the second attribute.

\section{EXTENSIONS}

So far, the discussion has been confined to stimuli defined by two attributes only. However, the general case
(

(a)
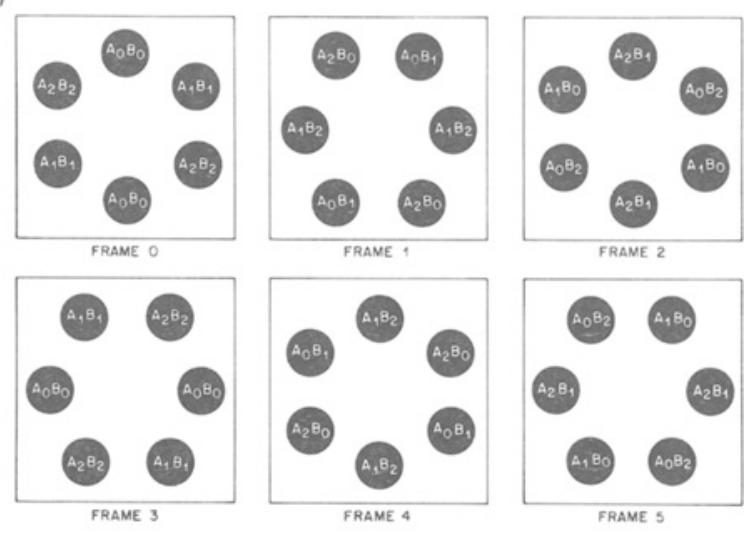

(b)

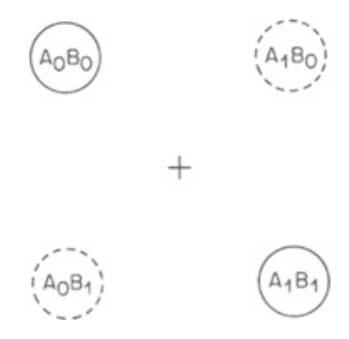

Figure 5. (a) Six frames in a sequence in which simultaneous clockwise and counterclockwise motion is elicited by matching attributes $A$ and $B$, respectively. Notice that this sequence is periodic with period 6. Thus it can be repeated to produce continuous movement of arbitrarily long duration. (b) A two-frame sequence for a multiattribute bistable quartet. The elements denoted by solid and dashed circles are displayed in frames 0 and 1, respectively. 


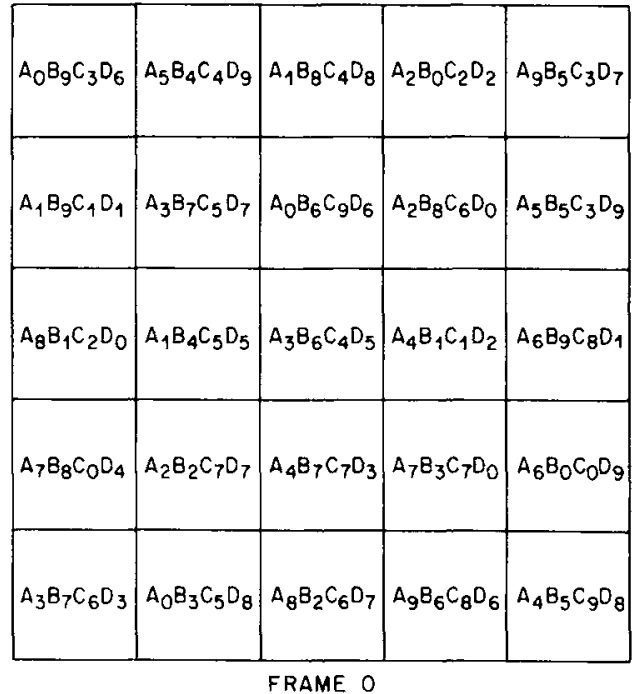

Figure 6. Two frames of a sequence in which motion is elicited by matching attributes $A, B, C$, and $D$. In this case, attributes $A, B, C$, and $D$ produce the perception of movement to the left, to the right, downward, and along the upper-right diagonal, respectively, as can be verified by inspecting the subscripts of each attribute.

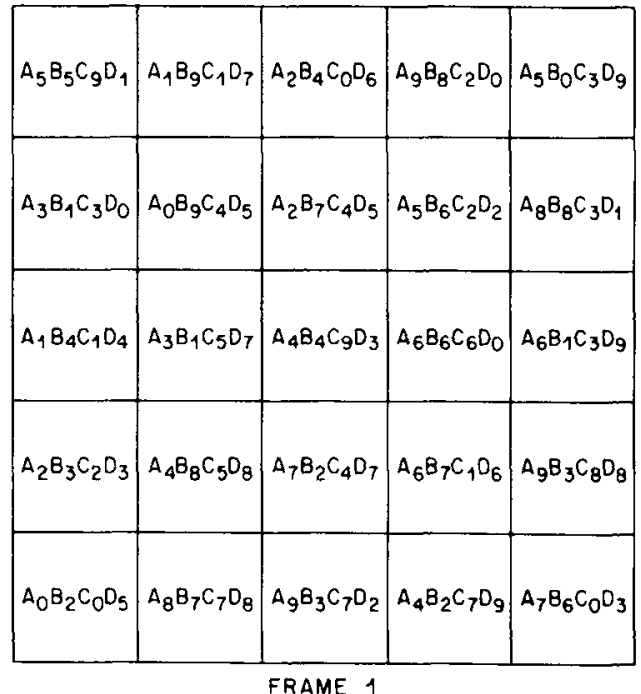

allows a combination of many attributes, subgroups of which can be arranged to produce independent motions. For instance, in a group of five attributes, the first three can be arranged so as to elicit leftward movement while the remaining two elicit rightward movement.

Another area for generalization is the choice of $\Delta x$. There is nothing that prevents one from using $\Delta x \neq x_{0} / 2$, particularly in the following situation. Suppose that, with $\Delta x=x_{0} / 2$, attribute $A$ has prevailed over $B$ in carrying motion with stimuli where $A$ and $B$ oppose each other. Then we can bias $\Delta x$ such that attributes $A$ and $B$ will elicit a "balanced" motion perception. In this way, it is possible to quantify the relative strength of pairs of attributes. Such a quantitative pairwise comparison can lead to a ranking of attributes with respect to "motion strength."

The concept of simultaneous multiattribute motion can be applied to other researchers' stimuli. As an example, Green (1986) used circularly arranged stimuli with $\Delta x=$ $x_{0} / 2$ that produced counterclockwise or clockwise movement due to a single attribute's configuration with $N=2$. The extended form of Green's stimuli with two attributes and $N=3$ is shown in Figure 5, where motion is elicited in the clockwise and counterclockwise directions by attributes $A$ and $B$, respectively. Of course, an arbitrary number of attributes can be used in this case, just as in the original form of our class of stimuli. An additional example is shown in Figure 5b, which offers a generalization to Ramachandran and Anstis's (1986) "bistable quartet." If frames $\mathbf{0}$ and 1 are displayed alternatively, then attributes $A$ and $B$ will tend to favor up-and-down and right-and-left movement, respectively.

Finally, our work with this type of stimuli has led us to create, along with Jean Lorenceau of the Laboratorie de Psychologie Expérimentale, the sequence shown in Figure 6. For simplicity, a display of only $5 \times 5$ "blocks" and two frames is shown. Notice that a spatially twodimensional $(x, y)$ arrangement for each frame is illus- trated. Each block is characterized by an arbitrary number of attributes $A, B, C, \ldots, Z$. Four attributes are shown in Figure 6, each of which can take on random values for any pair $(x, y)$ from among 10 different choices $(0-9)$. Inspection of frames 0 and 1 in Figure 6 reveals that attributes $A, B, C$, and $D$ have been matched to elicit simultaneous motion to the left, to the right, downward, and upward, respectively. Preliminary experiments with these stimuli indicate that they offer an additional degree of freedom by allowing motion in the $x-y-t$ space rather than just the $x-t$ space.

\section{CONCLUSION}

A new class of visual stimuli has been presented, whose main innovation is that it allows each of the multiple attributes characterizing the apparently moving elements to be properly arranged in the $x-t$ (or $x-y-t$ ) space so as to elicit ambiguous or unambiguous motion perception. These stimuli have been employed in psychophysical experiments for assessing the relative strength of attributes such as color, orientation, luminance, spatial frequency, binocular disparity, and polarity in producing motion. We believe that this class of stimuli offers a valuable tool for further experiments in motion perception.

\section{REFERENCES}

Adelson, E. H., \& BerGen, J. R. (1985). Spatiotemporal energy models for the perception of mation. Joumal of the Optical Sociery of America, 2A, 284-299.

ANsTrs, S. M. (1980). The perception of apparent movement. Philosophical Transactions of the Royal Society of London Series B, 290, 153-168.

Burt, P., Speruing, G. (1981). Time, distance and feature tradeoffs in visual apparent motion. Psychologial Review, 88, 171-195.

CAELL, T. (1986). Digital image-processing techniques for the display of images and modeling of visual perception. Behavior Research Methods, Instruments, \& Computers, 18, 493-506.

Cavanagh, P., \& ANSTIS, S. M. (1986). Do opponent-color channels 
contribute to motion? Supplement to Investigative Ophthalmology \& Visual Science, 27, 191.

Cavanagh, P., Anstis, S. M., \& Mather, G. (1984). Screening for color blindness using optokinetic nystagmus. Investigative Ophthalmology \& Visual Science, 25, 463-466.

Cavanagh, P., Favreau, O. E. (1985). Color and luminance share a common motion pathway. Vision Research, 25, 1595-1601.

Cavanagh, P., Tyler, C. W., \& Favreau, O. E. (1984). Perceived velocity of moving chromatic gratings. Journal of the Optical Society of America, 2A, 893-899.

Chubb, C., Sperling, G. (1987). Drift-balanced random stimuli: A general basis for studying non-Fourier motion mechanisms. Supplement to Investigative Ophthalmology \& Visual Science, 28, 233.

Daugman, J. (1984). Spatial visual channels in the Fourier plane. Vision Research, 24, 891-910.

Gorea, A., \& Papathomas, T. V. (1988a). The concept of "veto" attributes in motion perception. Investigative Ophthalmology \& Visual Science, 29, 265.

Gorea, A., Papathomas, T. V. (1988b). Form and surface attributes in motion perception studied with a new class of stimuli: $A$ basic asymmetry. Manuscript submitted for publication.

Gorea, A., PAPAThomas, T. V. (1988c). Motion processing in chromatic and achromatic visual pathways. Manuscript submitted for publication.

Green, M. (1986). What determines correspondence strength in apparent motion? Vision Research, 26, 599-607.

Green, M., ODom, V. (1986). Correspondence matching in apparent motion: Evidence for three-dimensional spatial representation Science, 233, 1427-1429.

HochberG, J. (1978). Perception (2nd ed., pp. 205-206). Englewood Cliffs, NJ: Prentice-Hall.

HochBERG, J., \& Brooks, V. (1974). The integration of successive cinematic views of simple scenes. Bulletin of the Psychonomic Society, 4, 263.

Kolers, P. A. (1972). Aspects of motion perception (pp. 46-58). Oxford, England: Pergamon Press.

Kolers, P. A., \& Pomerantz, J. R. (1971). Figural changes in apparent motion. Journal of Experimental Psychology, 87, 99-108.

Kool, F. L., DE Valois, R. L., \& WYman, T. K. (1988). Perceived direction of moving plaids. Investigative Ophthalmology \& Visual Science, 29, 265.

MiChAEL, C. R. (1985). Laminar segregation of color cells in the monkey striate cortex. Vision Research, 25, 415-423.

MoRELAND, J. D. (1980). Spectral sensitivity measured by motion photometry. In G. Verriest (Ed.), Color deficiencies VI (pp. 61-66). The Hauge, The Netherlands: Junk.
NAvon, D. (1976). Irrelevance of figural identity for resolving ambiguities in apparent motion. Joumal of Experimental Psychology: Human Perception \& Performance, 2, 130-138.

Pantle, A., \& Sexuler, R. (1969). Contrast response of human visual mechanisms sensitive to orientation and motion. Vision Research, 9 , $397-406$.

Papathomas, T. V., Gorea, A., Julesz, B., \& Chang, J. J. (1988). The relative strength of depth and orientation in motion perception. Investigative Ophthalmology \& Visual Science, 29, 401.

Ramachandran, V. S., \& Anstis, S. M. (1986). The perception of apparent motion. Scientific American, 254, 102-109.

Ramachandran, V. S., Ginsburg, A. P., \& Anstis, S. M. (1983). Low spatial frequencies dominate apparent motion. Perception, 12, 457-461.

Ramachandran, V. S., \& Gregory, R. L. (1978). Does color provide an input to human motion perception? Nature, 275, 55-56.

SPERLING, G. (1976). Movement perception in computer-driven visual displays. Behavior Research Methods \& Instrumentation, 8, 144-151.

Sperling, G., van Santen, J. P. H., \& Burt, P. J. (1985). Three theories of stroboscopic motion detection. Spatial Vision, 1, 47-56.

Thompson, P. (1982). Perceived rate of movement depends on contrast. Vision Research, 22, 377-380.

Troscianko, T. (1987). Perception of random-dot symmetry and apparent movement at and near isoluminance. Vision Research, 27, 547-554.

Ullman, S. (1979). The interpretation of visual motion. Cambridge, MA. MIT Press.

UlLmaN, S. (1980). The effect of similarity between line segments on the correspondence strength in apparent motion. Perception, 9, 617-626.

van SAnTEN, J. P. H., \& Sperling, G. (1985). Elaborated Reichardt detectors. Journal of the Optical Society of America, 2A, 300-321.

Watson, A. B. (1986). Apparent motion occurs only between similar spatial frequencies. Vision Research, 26, 1727-1730.

Watson, A. B., \& Ahumada, A. J., JR. (1983). A look at motion in the frequency domain. (NASA Tech. Memo No. TM-84352). Washington, DC: NASA.

ZEKI, S. M. (1983). The distribution of wavelength and orientation selective cells in different areas of monkey visual cortex. Proceedings of the Royal Society of London Series B, 217, 449-470.

(Manuscript received April 21, 1988; revision accepted for publication August 2, 1988.) 